\title{
Impact of type 2 Diabetes and Metformin use on Vitamin B12 Associated Biomarkers - an Observational Study
}

\author{
Authors \\ Corina Metaxas ${ }^{1}$, Chantal Zurwerra ${ }^{1}$, Gottfried Rudofsky ${ }^{2}$, Kurt E. Hersberger ${ }^{1}$, Philipp N. Walter ${ }^{1,3}$
}

\section{Affiliations}

1 Pharmaceutical Care Research Group, University of Basel, Switzerland

2 Department of Medicine, Kantonsspital Olten, Switzerland

3 Institute of Laboratory Medicine, Solothurn Hospitals, Switzerland

Key words

T2DM, metformin, vitamin B12 deficiency, vitamin B12, holotranscobalamine

received 07.08 .2017

revised 29.09.2017

accepted 05.10 .2017

\section{Bibliography}

DOI https://doi.org/10.1055/s-0043-120760

Published online: 2.2.2018

Exp Clin Endocrinol Diabetes 2018; 126: 394-400

(c) J. A. Barth Verlag in Georg Thieme Verlag KG Stuttgart .

New York

ISSN 0947-7349

\section{Correspondence}

Corina Metaxas

Pharmaceutical Care Research Group

Pharmazentrum

Klingelbergstrasse 50

$\mathrm{CH}-4056$ Basel

Tel.: +41(0)6120719 44, Fax: +41(0)61 2071428

corina.metaxas@unibas.ch

\section{ABSTRACT}

Aims Assessment of the impact of type 2 diabetes (T2DM) and metformin use on vitamin B12 (VB12) associated biomarkers and their suitability to represent VB12 supply.

Methods Differences of VB12, holotranscobalamine (HoloTc), the biologically active fraction $(\% \mathrm{AB} 12)=$ HoloTc $/ \mathrm{VB} 12 * 100$ and homocystein (Hcy) were analysed i) among diabetic outpatients with (DMMet + ) and without metformin use (DMMet-) and ii) in comparison to an external non-diabetic reference group with low VB12 (<200 pmol/L).

Results VB12 associated biomarkers were distributed equally between DMMet+ $(n=29,58 \%)$ and DMMet- $(n=21,42 \%)$. Significant differences in \%AB12 in diabetic patients with low VB12 $(n=19)$ compared to the non-diabetic reference group $(n=31)$ were found. Higher \%AB12 was associated with diabetes. Hcy levels were significantly associated with age, folic acid level, renal function and HoloTc but not with VB12.

Conclusions In T2DM patients with low VB12, \%AB12 was confirmed as being higher in comparison to nondiabetic patients. The effect was not clearly attributable to metformin use. HoloTc was unaffected by the lowering of VB12 and significantly associated with the functional marker Hcy. Both findings support the use of HoloTc for the assessment of VB12 supply in diabetic patients.

\section{Introduction}

Recently, an increased frequency of VB12 deficiency among T2DM patients has been documented by several cross sectional studies and case reports [1]. Clinically, VB12 deficiency in adults may result in nonspecific symptoms such as tiredness, loss of appetite, hematologic manifestations (megaloblastic anemia), neurologic symptoms (e. g., polyneuropathy, ataxia), as well as symptoms of a psychiatric nature (e. g., depression) [2,3]. Additionally, cardiovascular manifestations associated with hyperhomocysteinemia were mentioned [4-7].
Clinical symptoms of VB12 deficiency in T2DM are comparable to those in the general population. Worsening of diabetic neuropathy has been described among patients with co-existing vitamin B12 deficiency. Furthermore, VB12 replacement has been shown to cause symptomatic improvement, reduction in pain, and paranesthesia among patients with severe diabetic neuropathy [1], suggesting that functional VB12 deficiency in T2DM patients is clinically significant. Sensory polyneuropathy caused by VB12 deficiency mimics diabetic neuropathy [8]. Overlapping symptoms between T2DM and VB12 deficiency may complicate its clinical suspicion (or diagnosis). Biochemically, VB12 deficiency is charac- 
terized by subnormal to borderline VB12 values in serum $(<148-$ $221 \mathrm{pmol} / \mathrm{L}$ ) [9]. Holotranscobalamine (HoloTc) is the bioactive form of VB12 and makes up to $20 \%$ of the total vitamin B12 concentration in the human body [10]. It has been discussed as a more specific and sensitive marker of VB12 deficiency [11-13]. Broad ranges of cut-off points for HoloTc have been described as <20-50 pmol/L [14]. Functional VB12 deficiency is characterized by elevated homocysteine (Hcy) and/or methylmalonic acid (MMA) levels [9].

Additionally, the clinical biochemistry of VB12 is influenced by diabetes and its treatment. Systematic observations in clinical trials as well as biochemical studies in animals raised questions on possible interactions between diabetes, metformin treatment, and VB12 metabolism. It has been proposed that the increased oxidative stress in diabetes is involved in the pathogenesis of functional VB12 deficiency [15]. Treatment of T2DM patients with metformin has been reported with reductions of $10-20 \%$ in plasma VB12 levels [16-20]. Metformin may impair VB12 absorption and thereby induce VB12 deficiency $[20,21]$. One study described the correlation of cumulative metformin dose, low VB12 levels, and clinically more severe peripheral neuropathy [8]. Given the widespread use of metformin as first line treatment for patients with diabetes and normal kidney function, its effect on VB12 metabolism is remarkable [22]. Metformin is a biguanide. Its mechanism of action primarily involves decreasing hepatic glucose production and increasing glucose uptake [23]. Because VB12 deficiency is a reversible cause of demyelinating nervous system disease and bone marrow failure, its early detection and treatment are important [3]. Therefore, the identification of metformin as a risk factor for the development of VB12 deficiency is important and the evaluation of VB12 status is recommended [24]. However, there is reasonable doubt whether the reduction of VB12 levels reflects a true decrease in VB12 supply. A recent study in patients with diabetes ( $>65$ years old) which were treated for 3 months with metformin showed that the inactive part of VB12 bound to haptocorrin is reduced in metformin-treated patients but not in the control group (non-significantly) [25]. Additionally, a study in rats found that metformin treatment increases liver accumulation of VB12 thereby resulting in decreased circulating VB12 and kidney accumulated VB12 [26]. The same authors found a significant reduction of serum VB12 but not HoloTc after 6 months of metformin treatment in women with polycystic ovarian syndrome (PCOS) [27]. These findings raise the question of whether low serum VB12 observed in patients treated with metformin actually reflects VB12 deficiency and rather supports the hypothesis of altered metabolism of VB12 in metformintreated patients.

In this study, the effect of metformin treatment on VB12 status as reflected by total VB12 and HoloTc in T2DM patients is investigated. In particular, the \%AB12 in T2DM patients with and without metformin treatment was compared and interactions between VB12, HoloTc, and homocysteine were analysed. Additionally, the impact of diabetes itself on VB12 associated biomarkers at low VB12 levels is investigated in a subgroup of patients with diabetes in comparison to an external reference group of non-diabetic patients.

\section{Materials and Methods}

This was an observational cross-sectional trial approved by the ethics committee of northwestern Switzerland (EKNZ), and was registered at ClinicalTrials.gov NCT02111967. The study was conducted in accordance with the Declaration of Helsinki and correspondent to International Conference on Harmonization Good Clinical Practice (ICH-GCP) guidelines. All patients gave written informed consent. Patients for the external reference group were included from a previous study (ClinicalTrials.gov NCT01832129). The primary hypothesis was that VB12 would be lower in T2DM patients treated with metformin while HoloTc levels would not differ compared to T2DM patients without metformin treatment.

\section{Setting}

The study included adults with type 2 diabetes and an external reference group of nondiabetic and metformin-naïve patients. Patients with T2DM were classified as metformin users (DMMet+) if they were treated with metformin for at least 6 months or as metformin-naïve patients (DMMet-), when no prior use of metformin was reported ( $\vee$ Fig. 1; A). Participants with newly initiated metformin (<6 months usage), concurrent intake of preparations containing VB12 (in the last 3 months), diagnosis of transcobolamin transporter defect, diagnosis of vitamin B6 deficiency, diagnosis of liver disease with CHILD-PUGH scores B and C, acute hepatitis, and diagnosis of alcohol abuse (defined as a diagnosed condition or by self-reported daily intake of alcohol), diagnosis of renal insufficiency stadium III, IV, and V (KDOQI), acute renal diseases or renal function below $60 \mathrm{ml} / \mathrm{min}$ according to the Cockroft-Gault equation were excluded from the study. For the reference group, data from patients from a previous study were used [28]. Out of 37 patients, 4 were excluded due to an alcohol use disorder $(n=4)$.(

\section{Patient recruiting}

Patients with diabetes with (DMMet +) and without metformin treatment (DMMet-) were recruited during a routine visit at their diabetes specialist ( $\triangleright$ Fig. 1; sample A). Patients with diabetes and serum VB12 levels below 200 pmol/L were classified as VB12 deficient (LVB12-DM). The external reference group of non-diabetic patients with low VB12 levels (LVB12-Ref) was established from a previously published study that recruited outpatients during a routine visit to their general practitioner. If patients had a diagnosis of diabetes, they were not excluded but assigned to the LVB12-DM Group ( $\triangleright$ Fig.1, sample B).

\section{Questionnaires and physical examinations}

Patients were asked to fill in a questionnaire about their nutrition, co-medication, and demographics. Patients with type 2 diabetes were screened for neuropathy signs using the Neuropathy Symptom Score (NSS) and the Neuropathy Disability Score (NDS) [29].

\section{Pharmacological biomarker}

All venous blood samples were analyzed for VB12 (Beckman Coulter ${ }^{\circledR}$ DxC 860i), HoloTc (Abbott Architect ${ }^{\circledR}$ i2000SR), and Hcy (Roche Cobas $(6000)$. The biologically active fraction (\%AB12) was calculated by dividing HoloTc/VB12* 100. Blood cell count was de- 
Patient recruitment T2DM

$\mathrm{n}=50$

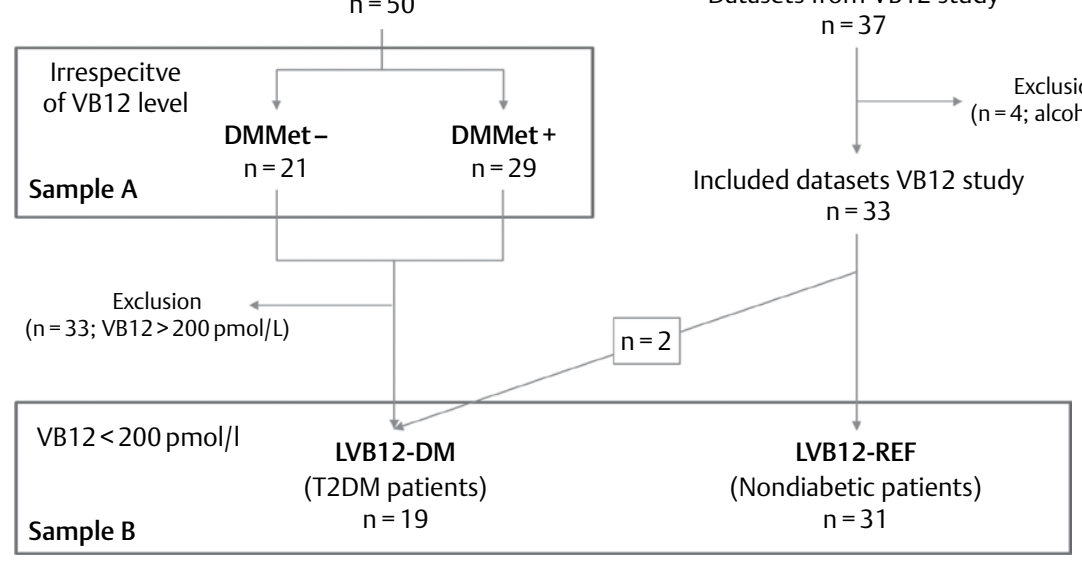

Fig. 1 Sample A) Patients recruited by the diabetologist. Comparison of VB12, HoloTc and \% AB12 between DMMet + and DMMet- and assessment of associations between VB12, HoloTc, Hcy and severity of neuropathy. Sample B) Patients with low VB12 $(<200$ pmol/L) recruited by diabetologist and general practitioners. Comparison of VB12, HoloTc and \% AB12 between LVB12-DM and LVB12-Ref.

termined on a Beckman Coulter DxH 800. Cut-off values defining VB12 deficiency were VB12 <200 pmol/L, HoloTc $<37 \mathrm{pmol} / \mathrm{L}$ and $\mathrm{Hcy}>15 \mu \mathrm{mol} / \mathrm{L}$. Blood samples of patients with VB12 levels below $200 \mathrm{pmol} / \mathrm{L}$ were re-analyzed for VB12 on the Roche Cobas ${ }^{\circledR} 6000$ to directly compare the results with the external reference group.

\section{Study size}

Based on published data, patients with diabetes were assumed to display VB12 levels of approximately $400 \mathrm{pmol} / \mathrm{l}$, with an estimated standard deviation of $250 \mathrm{pmol} / \mathrm{l}$ (62.5\%) [30]. In this study, $40.1 \%$ of patients had consumed VB12 supplements; therefore, we concluded that the standard deviation among patients without VB12 supplements was approximately half of the observed standard deviation (30\%). A reduction in plasma VB12 levels of $20-24 \%$ is described in the scientific literature [25]. Since we expected a reduction in levels, a one-tailed test was applicable. Based on a reduction of VB12 levels of $24 \%$, a total of 50 samples had had to be analysed to ensure that the $80 \%$ confidence interval includes the true difference of means with a $\alpha$-significance level of $5 \%$.

\section{Statistical analysis}

Values are given as medians, with quartiles and percentages where appropriate. Frequencies were analyzed using Chi-square tests or Fisher's test. The Mann-Whitney test was used to compare numerical variables between two groups. Bivariate correlation was performed when association between two scaled variables was assessed. A p-value $\leq 0.05$ was considered significant. Multiple linear regression analyses were conducted to calculate i) the relative impact of VB12 and HoloTc on Hcy in relation to age, renal function assessed as estimated Glomerular filtration rate (eGFR) according to the Cockroft-Gault equation, and folic acid levels; and ii) the relative impact of diabetes, age, $\mathrm{BMI}$, renal function and folic acid levels on the biologically active fraction $\%$ AB12 = HoloTc/VB12 * 100 using a stepwise method to include the independent variables. The statistical procedures were performed with SPSS statistical software Version 24 (SPSS Inc., Chicago, II, USA).

\section{Results}

\section{Participants}

Between March 2014 and August 2014, 50 outpatients with diabetes were recruited within the endocrinology unit. Recruitment and patient characteristics of the external reference group have been described elsewhere [28]. Two of the 37 recruited subjects in this study had diabetes and were thus integrated in the respective subgroup for the analysis of VB12 associated biomarkers in diabetes (> Fig. 1). Patient characteristics of T2DM patients (29 DMMet+;21 DMMet-) were equally distributed (॰ Table 1).

\section{VB12 associated biomarker levels in diabetes pa- tients (sample $A$ )}

DMMet+ versus DMMet-

$\mathrm{VB}_{12}$ serum levels and HoloTc levels were slightly lower in DMMet + patients compared to DMMet-, but not significantly (VB12: $-10.4 \%$; HoloTc: $-9.2 \%$ Table 2). The \%AB12, Hcy and blood count levels were equally distributed between the two groups ( $\triangleright$ Table 2). Out of 50 patients, 17 (34\%) had a VB12 value below 200 pmol/L (11 DMMet+ (39.3\%) and 6 DMMet- (28.6\%); Fishers-test: 0.55), 3 (6\%) had a HoloTc value below $37 \mathrm{pmol} / \mathrm{L}$ (3 DMMet + (10.3\%) and 0 DMMet- (0\%); Fishers-test: 0.25$)$ and $16(32 \%)$ had a Hcy level above $15 \mu \mathrm{mol} / \mathrm{L}$ (10 DMMet + (34.5\%) and 6 DMMet- (28.6\%); Fishers-test: 0.75$)$ (data not shown).

Correlations of $\mathrm{VB}_{12}$ associated biomarkers

Significant correlations $(p<0.05)$ of VB12 levels with age $(r=-0.42)$ and $\mathrm{Hcy}(r=-0.37)$ were found for the entire study population, as well as with metformin dose $(r=-0.43)$, and renal function $(r=-0.41)$ in DMMet + patients. For HoloTc, significant correlations 
- Table 1 Patient characteristics of sample A and potential risk factors for the development of VB12 deficiency of DMMet+ group and DMMetgroup.

\begin{tabular}{|l|c|c|c|}
\hline & $\begin{array}{c}\text { DMMet+ } \\
\text { (n=29) }\end{array}$ & $\begin{array}{c}\text { DMMet- } \\
\text { (n=21) }\end{array}$ & p-value \\
\hline Age (years) & $60(53 / 70)$ & $64(55 / 72)$ & 0.42 \\
\hline Women (\%) & 44.8 & 38.1 & 0.43 \\
\hline BMI (kg/m2) & $30.9(24.9 / 40.6)$ & $33.8(30.2 / 37.8)$ & 0.33 \\
\hline $\begin{array}{l}\text { Years with } \\
\text { diabetes }\end{array}$ & $7(4 / 15)$ & $12.5(5.5 / 20)$ & 0.10 \\
\hline HbA1C (\%) & $7.7(6.7 / 8.4)$ & $8.7(6.9 / 9.8)$ & 0.17 \\
\hline NSS+NDS & $6.5(2.8 / 11.3)$ & $11(5.3 / 14.8)$ & 0.09 \\
\hline $\begin{array}{l}\text { eGFR (Cockroft } \\
\text { Gault, ml/min) }\end{array}$ & $115(78.4 / 160.4)$ & $116(74.7 / 157)$ & 0.78 \\
\hline $\begin{array}{l}\text { Proton-pump } \\
\text { inhibitors use (\%) }\end{array}$ & 6.9 & 19.0 & 0.22 \\
\hline $\begin{array}{l}\text { Antacid and } \mathrm{H}_{2} \\
\text { Blocker use (\%) }\end{array}$ & 0 & 0 & - \\
\hline Vegetarians (\%) & 6.9 & 0.0 & 0.33 \\
\hline Vegans (\%) & 0.0 & 0.0 & - \\
\hline $\begin{array}{l}\text { Parameters are given as median and 25/75 percentiles if not } \\
\text { otherwise indicated. }\end{array}$ & & \\
\hline
\end{tabular}

- Table 2 VB12 associated biomarker and blood count levels of DMMet+ and DMMet- groups.

\begin{tabular}{|l|c|c|c|}
\hline & DMMet+ (n=29) & DMMet- (n=21) & p-value \\
\hline $\begin{array}{l}\text { VB12 (pmol/L), } \\
\text { Beckman }\end{array}$ & $224(163 / 263)$ & $250(190 / 344)$ & 0.19 \\
\hline HoloTc $(\mathrm{pmol} / \mathrm{L})$ & $79.9(46 / 102)$ & $88(68 / 111)$ & 0.09 \\
\hline$\% A B 12$ & $30.1(25.0 / 40.8)$ & $35.4(29.5 / 39.6)$ & 0.32 \\
\hline $\mathrm{Hcy}(\mu \mathrm{mol} / \mathrm{L})$ & $12.9(11.3 / 17.5)$ & $11.8(10.5 / 19.3)$ & 0.55 \\
\hline $\mathrm{Hb}(\mathrm{g} / \mathrm{L})$ & $142(133 / 154)$ & $145(139 / 152)$ & 0.71 \\
\hline $\mathrm{MCV}(\mathrm{fL})$ & $90(88 / 93)$ & $93(88 / 96)$ & 0.12 \\
\hline MCH $(\mathrm{pg})$ & $30(29 / 31)$ & $31(30 / 32)$ & 0.07 \\
\hline $\begin{array}{l}\text { Parameters are given as median and 25/75 percentiles if not } \\
\text { otherwise indicated. }\end{array}$ \\
\hline
\end{tabular}

were limited to Hcy $(r=-0.44)$ and metformin dose $(r=-0.47)$ in DMMet+ patients. High Hcy levels were associated with high age $(r=0.35)$, low renal function $(r=-0.48)$, and low folic acid levels $(r=-0.36)$ in the entire study population. Mean daily metformin dose in DMMet + group was $1.9 \mathrm{~g} \pm 0.6 \mathrm{~g}$ (range: $1.0 \mathrm{~g}-3.0 \mathrm{~g}$ ).

Prediction of Hcy levels

In a multiple linear regression model, a significant association of Hcy with age $(\beta=0.325 ; p=0.029)$, folic acid $(\beta=-0.359$; $p=0.003)$, HoloTc levels $(\beta=-0.300 ; p=0.01)$, and renal function expressed as eGFR by Cockroft-Gault $(\beta=-0.343$; $p=0.014)$ was found in all DM patients $\left(r^{2}=0.539 ; p=0.022\right)$, while no significant association of VB12 with Hcy was observed.

VB12 associated biomarker levels according to the severity of neuropathy

Neither VB12, HoloTc, nor Hcy differed significantly between patients with mild, moderate, and severe symptoms of neuropa- thy ( $\triangleright$ Fig. 2). Age and diabetes duration were significantly higher in patients with severe neuropathy compared to patients with mild neuropathy.

\section{Analysis in patients with low VB12 levels (sample B)} LVB12-DM versus LVB12-REF

Diabetes patients with low VB12 (LVB-DM) were compared with a non-diabetic reference group (LVB-REF). In the LVB-REF group, a trend towards higher HoloTc level and significantly higher \%AB12 was found ( $>$ Table 3). Patients in the reference group were younger and had a significantly lower mean BMI (

\section{Prediction of \%AB12 in patients with low VB12}

A multiple linear regression showed a significant association of \%AB12 with diabetes $\left(\beta=-0.336 ; p=0.02 ; r^{2}=0.092 ; p=0.023\right)$, whilst no significant associations of the \%AB12 with age, $B M I$, renal function expressed as eGFR (Cockroft-Gault) or folic acid levels were observed.

\section{Discussion}

In our study, neither VB12, HoloTc or \%AB12 differed between T2DM patients regardless of metformin treatment. Median VB12 and HoloTc levels were within the normal range in both groups. Therefore, the primary hypothesis that VB12 levels would differ while HoloTc levels would be indifferent between T2DM patients with or without metformin treatment had to be rejected. Metformin treatment alone did not explain the altered VB12 metabolism as reflected by VB12 and HoloTc serum levels in all T2DM patients, as suggested by the literature [26,27]. Nevertheless, the proportion of patients in the DMMet+ group with low VB12, low HoloTc, or high Hcy was higher compared to DMMet- group (not significant). Additionally, metformin dosage did negatively correlate with VB12 and HoloTc levels. These findings suggest that metformin may contribute to VB12 deficiency.

Further analysis focused on VB12-deficient subgroups and included non-diabetic patients. In this sample (B), a significant difference of the \%AB12 was observed and confirmed by multiple regression analysis. However, the model explained only $9.2 \%$ of the variance observed. These results suggest that VB12 metabolism is affected by diabetes itself, as well as by other factors which were not included in the model. It has been proposed that duration of VB12 deficiency and causes of VB12 deficiency play a major role in the VB12/HoloTc ratio [31], such as liver diseases [32], in particular alcoholism [33]. In this study, patients with liver diseases and/ or alcoholism were excluded. Duration of a VB12 deficiency can hardly be assessed in an observational study; therefore, interventional studies might be favorable to continue research on VB12 metabolism. Multiple regression analysis showed that T2DM in general had an impact on \%AB12. Further observations regarding the impact of metformin in T2DM patients with low VB12 had a high variance (data not shown) due to the relatively small sample (17 T2DM patients; 11 DMMet+ and 6 DMMet-) and therefore were not taken into account. Thus, larger studies may be necessary to differ whether the altered VB12 metabolism is attributable to diabetes in general, or specifically to metformin use. 


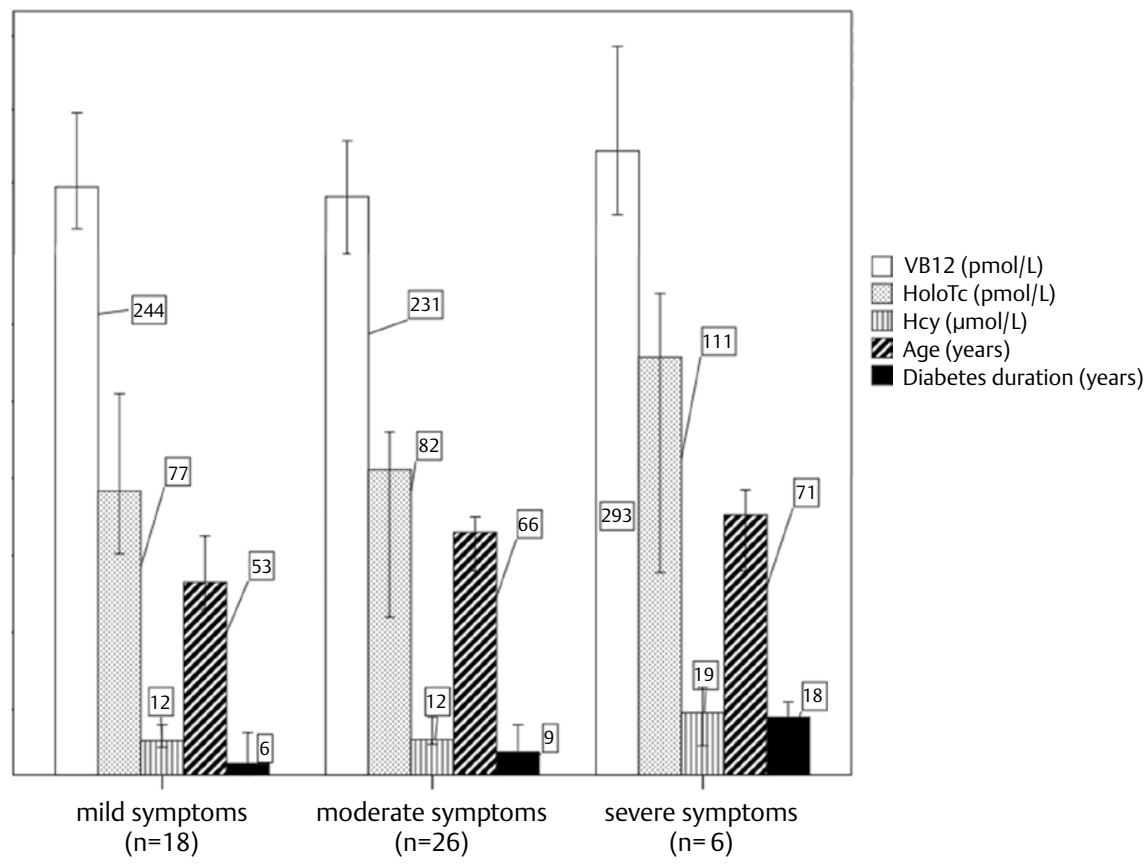

- Fig. 2 Median levels with 95\% confidence intervals of VB12 (pmol/L), HoloTc (pmol/L), Hcy ( $\mu \mathrm{mol} / \mathrm{L}$ ), Age (years) and diabetes duration (years) in patients with mild symptoms, moderate and severe symptoms of neuropathy.

- Table 3 VB12 associated biomarker and blood count levels of sample B.

\begin{tabular}{|c|c|c|c|}
\hline & LVB-DM $(n=19)$ & LVB-REF $(n=31)$ & p-value \\
\hline Women (\%) & $50 \%$ & $71 \%$ & 0.22 \\
\hline Age (years) & $65(57 / 74)$ & $47(30 / 60)$ & 0.001 \\
\hline BMI (kg/m2) & $29.6(24.4 / 34.2)$ & $24.4(22.6 / 29.0)$ & 0.02 \\
\hline VitB12 (pmol/L), Roche & $162.0(140 / 178)$ & $164.0(149 / 177)$ & 0.75 \\
\hline HoloTc (pmol/L) & $53.2(42.0 / 78.1)$ & $43.0(32.1 / 62.2)$ & 0.08 \\
\hline$\% A B 12$ & $32.1(25.5 / 48.5)$ & $27.7(21.5 / 40.0)$ & 0.04 \\
\hline $\mathrm{Hcy}(\mu \mathrm{mol} / \mathrm{L})$ & $13.4(11.0 / 17.3)$ & $11.9(9.8 / 15.3)$ & 0.40 \\
\hline $\mathrm{HB}(\mathrm{g} / \mathrm{L})$ & $134(131 / 143)$ & $136(127 / 144)$ & 0.98 \\
\hline $\mathrm{MCV}(\mathrm{fL})$ & $90(87 / 93)$ & $92(90 / 96.0)$ & 0.74 \\
\hline
\end{tabular}

HoloTc has been proposed as a better marker to detect VB12 deficiency compared to serum VB12 $[34,35]$ in an aged population [36]. We found significant inverse correlations of VB12 and HoloTc with Hcy, a functional marker of VB12 deficiency. Although the effect was stronger between VB12 and Hcy compared to HoloTc and $\mathrm{Hcy}$, stepwise multiple regression analysis included HoloTc as independent variable to explain variance in Hcy levels and not VB12. Thus, HoloTc seems favorable compared to VB12 to predict hyperhomocysteinemia caused by VB12 deficiency in T2DM patients. Therefore, our results support the finding that HoloTc might be a better marker than VB12 to detect VB12 deficiency. Furthermore, regression analysis showed that in our sample, elevated Hcy was also explained by age, folate deficiency or renal insufficiency, thus compromising its value as an independent reference for VB12 deficiency. Other studies found inverse correlations between GFR and Hcy within patients with normal renal function also [37, 38]. Metab- olism of methylmalonic acid, another functional biomarker of VB12 deficiency, is not affected by vitamin B6 or folic acid. Therefore, measurement of methylmalonic acid might be more sensitive in detecting VB12 deficiency than Hcy. However, methylmalonic acid levels may be compromised in patients with reduced GFR, too [39].

Results from a previous study questioned whether low VB12 in metformin-treated patients with diabetes causes a true or functionally irrelevant cobalamin deficiency [27]. Interestingly, in our study, significant inverse correlations of Hcy with VB12 and HoloTc were exclusively found in DMMet+ patients. Even though VB12 levels were comparable within all DM patients, low VB12 and HoloTc levels did cause functional deficiency in DMMet+ but not in DMMet. An explanation for this finding might be that no true VB12 deficient DMMet- patients were included in our study, supported by the fact that no HoloTc below $37 \mathrm{pmol} / \mathrm{L}$ was found in DMMetgroup. Furthermore, our data suggest that metformin induces 
VB12 deficiency in a dose-dependent manner. Other studies also found associations between metformin dose and VB12 levels $[37,40]$. Thus, it is reasonable to screen patients treated with metformin for VB12 deficiency, as proposed [41]. In a future study, inclusion of anemic patients might help to clarify whether the elevation of functional markers results from VB12 deficiency or from impaired GFR, and whether low VB12 and/or HoloTc coincide with anemia.

VB12, HoloTc or Hcy levels did not differ in patients with mild, moderate or severe symptoms of neuropathy assessed with the NSS and NDS, while, well-established risk factors such as age and duration of diabetes differed between groups. While some studies showed associations between neuropathy, VB12 associated biomarkers, and metformin use $[8,42]$, others failed to find such correlations [43-45], making results controversial, overall. Controversy might exist because a range of different assessments for neuropathy and differences in study designs exist. The observed incidence of a VB12 deficiency in this study was high (34\%). Irreversible neurologic damage caused by VB12 deficiency is preventable through treatment $[3,8]$ at relatively low costs, and has few side effects. Therefore, screening for VB12 deficiency independently from diabetic neuropathy seems reasonable.

We acknowledge some limitations. First, our sample size calculation was based on bigger difference in VB12 levels between T2DM patients with and without metformin. However, the prevalence of patients having VB12 deficiency defined as VB12 below $200 \mathrm{pmol} / \mathrm{L}$ (34\% (DMMet+ (39.3\%) and 6 DMMet- (28.6\%)) we observed is comparable to what is described for elderly users [46, 47]. Therefore, differences in VB12 associated biomarkers may also be observed within all DM patients when more patients were included. Second, the assessment if HoloTc or VB12 might be better to detect VB12 deficiency was only based on a laboratory marker (Hcy levels). In this study, non-anemic patients were included and therefore clinical symptoms where not taken into account. Further studies should include anemic patients and assess methylmalonic acid.

\section{Conclusion}

The clinical biochemistry of VB12 in T2DM patients with scarce VB12 supply is modified in comparison to nondiabetic patients. This results in higher \%AB12 due to reduced VB12 levels. It needs to be clarified whether this effect is due to diabetes itself, metformin treatment, and/ or a combination of other heath related situations. Assessment of HoloTc seems favorable compared to VB12 to predict hyperhomocysteinemia caused by VB12 deficiency in T2DM patients. This may be a direct consequence of the modified \%AB12 in T2DM patients, which strengthens the recommendation to assess VB12 supply in clinical practice by measuring HoloTc. VB12, HoloTc and Hcy did not differ in patients with mild, moderate, or severe symptoms of neuropathy.

\section{Acknowledgements}

We would like to thank all participating patients and all participating general practitioners and their staff for patient recruitment. We thank Ursula Zoli (Aarelab, Olten) and the laboratory technicians at the Institute of Laboratory Medicine in Olten for their prac- tical contribution. We thank William Caddy for proofreading the manuscript.

Other information: No grants from any external funding body were received to conduct this study.

\section{Conflict of Interest}

The authors declare no conflicts of interest.

\section{References}

[1] Kibirige D, Mwebaze R. Vitamin B12 deficiency among patients with diabetes mellitus: Is routine screening and supplementation justified? Journal of diabetes and metabolic disorders 2013; 12: 17

[2] Dali-Youcef N, Andres E. An update on cobalamin deficiency in adults. QJM: Monthly Journal of the Association of Physicians 2009; 102: 17-28

[3] Stabler SP. Clinical practice. Vitamin B12 deficiency. The New England journal of medicine 2013; 368: 149-160

[4] Bergen C, Compher C. Total homocysteine concentration and associated cardiovascular and renal implications in adults. The Journal of cardiovascular nursing 2006; 21: 40-46

[5] Pawlak R. Is Vitamin B12 deficiency a risk factor for cardiovascular disease in vegetarians? American Journal of Preventive Medicine 2015; 48: e11-e26

[6] Nygard O, Nordrehaug JE, Refsum $\mathrm{H}$ et al. Plasma homocysteine levels and mortality in patients with coronary artery disease. The New England journal of medicine 1997; 337: 230-236

[7] Seshadri S, Beiser A, Selhub J et al. Plasma homocysteine as a risk factor for dementia and Alzheimer's disease. The New England journal of medicine 2002; 346: 476-483

[8] Wile D], Toth C. Association of metformin, elevated homocysteine, and methylmalonic acid levels and clinically worsened diabetic peripheral neuropathy. Diabetes care 2010; 33: 156-161

[9] Schrier SL. Diagnosis and treatment of vitamin B 12 and folic acid deficiency. uptodate (online, last update 09/2016) 2016;

[10] Stabler SP. Vitamin B12 deficiency. The New England journal of medicine 2013; 368: 2041-2042

[11] Hvas AM, Nexo E. Holotranscobalamin-a first choice assay for diagnosing early vitamin B deficiency? Journal of internal medicine 2005; 257: 289-298

[12] Nexo E, Hoffmann-Lücke E. Holotranscobalamin, a marker of vitamin B-12 status: analytical aspects and clinical utility. The American journal of clinical nutrition 2011; 94: 359S-365S

[13] Yetley EA, Pfeiffer CM, Phinney KW et al. Biomarkers of vitamin B-12 status in NHANES: A roundtable summary. The American journal of clinical nutrition 2011; 94: 313S-321S

[14] Aparicio-Ugarriza R, Palacios G, Alder M et al. A review of the cut-off points for the diagnosis of vitamin B12 deficiency in the general population. In, Clinical Chemistry and Laboratory Medicine (CCLM); 2015: 1149

[15] Solomon LR. Diabetes as a cause of clinically significant functional cobalamin deficiency. Diabetes care 2011; 34: 1077-1080

[16] Carlsen SM. [Metformin and vitamin B 12 deficiency]. Tidsskrift for den Norske laegeforening: tidsskrift for praktisk medicin, ny raekke 2007; 127: 764 author reply 764 
[17] Sahin M, Tutuncu NB, Ertugrul D et al. Effects of metformin or rosiglitazone on serum concentrations of homocysteine, folate, and vitamin B12 in patients with type 2 diabetes mellitus. Journal of diabetes and its complications 2007; 21: 118-123

[18] Kilicdag EB, Bagis T, Tarim E et al. Administration of B-group vitamins reduces circulating homocysteine in polycystic ovarian syndrome patients treated with metformin: A randomized trial. Hum Reprod 2005; 20: 1521-1528

[19] Kancherla V, Elliott JL Jr., Patel BB et al. Long-term metformin therapy and monitoring for vitamin B12 deficiency among older veterans. Journal of the American Geriatrics Society 2017

[20] Liu Q, Li S, Quan H et al. Vitamin B12 status in metformin treated patients: Systematic review. PloS one 2014; 9: e100379

[21] Bauman WA, Shaw S, Jayatilleke E et al. Increased intake of calcium reverses vitamin B12 malabsorption induced by metformin. Diabetes care 2000; 23: 1227-1231

[22] Inzucchi SE, Bergenstal RM, Buse JB et al. Management of hyperglycaemia in type 2 diabetes, 2015: A patient-centred approach. Update to a Position Statement of the American Diabetes Association and the European Association for the Study of Diabetes. Diabetologia 2015; 58: 429-442

[23] Bosi E. Metformin--the gold standard in type 2 diabetes: What does the evidence tell us? Diabetes, obesity \& metabolism 2009; 11: (Suppl 2): $3-8$

[24] de Jager J, Kooy A, Lehert P et al. Long term treatment with metformin in patients with type 2 diabetes and risk of vitamin B-12 deficiency: randomised placebo controlled trial. BM] 2010; 340: c2181

[25] Leung S, Mattman A, Snyder F et al. Metformin induces reductions in plasma cobalamin and haptocorrin bound cobalamin levels in elderly diabetic patients. Clinical biochemistry 2010; 43: 759-760

[26] Greibe E, Miller JW, Foutouhi SH et al. Metformin increases liver accumulation of vitamin B12 - an experimental study in rats. Biochimie 2013; 95: 1062-1065

[27] Greibe E, Trolle B, Bor MV et al. Metformin lowers serum cobalamin without changing other markers of cobalamin status: A study on women with polycystic ovary syndrome. Nutrients 2013; 5: 24752482

[28] Metaxas C, Mathis D, Jeger C et al. Early biomarker response and patient preferences to oral and intramuscular vitamin B12 substitution in primary care: A randomised parallel-group trial. Swiss medical weekly 2017; 147: w14421

[29] Young M], Boulton AJ, MacLeod AF et al. A multicentre study of the prevalence of diabetic peripheral neuropathy in the United Kingdom hospital clinic population. Diabetologia 1993; 36: 150-154

[30] Reinstatler L, Qi YP, Williamson RS et al. Association of biochemical $\mathrm{B}(1)(2)$ deficiency with metformin therapy and vitamin $\mathrm{B}(1)(2)$ supplements: The National Health and Nutrition Examination Survey, 1999-2006. Diabetes care 2012; 35: 327-333

[31] Golding PH. Holotranscobalamin (HoloTC, Active-B12) and Herbert's model for the development of vitamin B12 deficiency: A review and alternative hypothesis. SpringerPlus 2016; 5: 668

[32] Baker H, Leevy CB, DeAngelis B et al. Cobalamin (vitamin B12) and holotranscobalamin changes in plasma and liver tissue in alcoholics with liver disease. Journal of the American College of Nutrition 1998; 17: $235-238$
[33] Fragasso A, Mannarella C, Ciancio A et al. Holotranscobalamin Is a Useful Marker of Vitamin B12 Deficiency in Alcoholics. The Scientific World Journal 2012; 2012: 128182

[34] Nexo E, Hvas AM, Bleie $\mathrm{O}$ et al. Holo-transcobalamin is an early marker of changes in cobalamin homeostasis. A randomized placebo-controlled study. Clinical chemistry 2002; 48: 1768-1771

[35] Obeid R, Herrmann W. Holotranscobalamin in laboratory diagnosis of cobalamin deficiency compared to total cobalamin and methylmalonic acid. Clinical chemistry and laboratory medicine: CCLM/FESCC 2007; 45: $1746-1750$

[36] Valente E, Scott JM, Ueland PM et al. Diagnostic accuracy of holotranscobalamin, methylmalonic acid, serum cobalamin, and other indicators of tissue vitamin $\mathrm{B}(1)(2)$ status in the elderly. Clinical chemistry 2011; 57: 856-863

[37] Sato $Y$, Ouchi K, Funase $Y$ et al. Relationship between metformin use, vitamin B12 deficiency, hyperhomocysteinemia and vascular complications in patients with type 2 diabetes. Endocrine journal 2013; 60: 1275-1280

[38] Wollesen F, Brattstrom L, Refsum $\mathrm{H}$ et al. Plasma total homocysteine and cysteine in relation to glomerular filtration rate in diabetes mellitus. Kidney international 1999; 55: 1028-1035

[39] Hannibal L, Lysne V, Bjorke-Monsen AL et al. Biomarkers and algorithms for the diagnosis of vitamin B12 deficiency. Frontiers in molecular biosciences 2016; 3: 27

[40] Ting R, Szeto C, Chan M et al. Risk factors of vitamin b12 deficiency in patients receiving metformin. Archives of internal medicine 2006; 166: $1975-1979$

[41] Kancherla V, Elliott JL Jr., Patel BB et al. Long-term metformin therapy and monitoring for vitamin B12 deficiency among older veterans. Journal of the American Geriatrics Society 2017; 65: 1061-1066

[42] Roy RP, Ghosh K, Ghosh M et al. Study of Vitamin B(12) deficiency and peripheral neuropathy in metformin-treated early Type 2 diabetes mellitus. Indian Journal of Endocrinology and Metabolism 2016; 20: 631-637

[43] Ahmed MA, Muntingh G, Rheeder P. Vitamin B12 deficiency in metformin-treated type-2 diabetes patients, prevalence and association with peripheral neuropathy. BMC Pharmacology \& Toxicology 2016; 17: 44

[44] Chen S, Lansdown AJ, Moat S] et al. An observational study of the effect of metformin on B12 status and peripheral neuropathy. The British Journal of Diabetes \& Vascular Disease 2012; 12: 189-193

[45] Russo GT, Giandalia A, Romeo EL et al. Diabetic neuropathy is not associated with homocysteine, folate, vitamin B12 levels, and MTHFR C677T mutation in type 2 diabetic outpatients taking metformin. Journal of endocrinological investigation 2016; 39: 305-314

[46] Wong CW. Vitamin B12 deficiency in the elderly: is it worth screening? Hong Kong medical journal = Xianggang yi xue za zhi/Hong Kong Academy of Medicine 2015; 21: 155-164

[47] Pflipsen MC, Oh RC, Saguil A et al. The prevalence of vitamin b12 deficiency in patients with type 2 diabetes: A cross-sectional study. The Journal of the American Board of Family Medicine 2009; 22: 528-534 